\title{
CONSTRUCTING 3-MANIFOLDS FROM GROUP HOMOMORPHISMS
}

\author{
BY WILLIAM JACO ${ }^{1}$
}

Communicated by R. H. Bing, April 8, 1968

1. Introduction. Let $S$ be a closed, orientable 2-manifold of genus $n>0$. Let $F_{1}$ and $F_{2}$ be free groups of rank $n$ and denote by $F_{1} \times F_{2}$ their direct product. Fix a point $s_{0}$ of $S$ and suppose $\eta_{1}, \eta_{2}$ are homomorphisms of $\pi_{1}\left(S, s_{0}\right)$ onto $F_{1}$ and $F_{2}$ respectively. The homomorphism

$$
\eta_{1} \times \eta_{2}: \pi_{1}\left(S, s_{0}\right) \rightarrow F_{1} \times F_{2}
$$

is called a splitting homomorphism of $\pi_{1}\left(S, s_{0}\right)$. Let $M$ be a closed, orientable 3-manifold. In [3] J. Stallings introduced a natural splitting homomorphism induced by a Heegaard splitting of $M$. The purpose of this paper is to announce that for any splitting homomorphism there is a closed, orientable 3-manifold $M$ and a Heegaard splitting of $M$ so that the induced splitting homomorphism is equivalent to the given splitting homomorphism. This is Theorem 4.1 of $\$ 4$. See $\$ 2$ for definitions.

It is shown in Theorem 4.2 that two conjectures made by J. Stallings in [3] are true if and only if Poincaré's Conjecture that any closed, simply-connected 3-manifold is a 3-sphere, is true. These conjectures appear in $\$ 4$ as Conjecture $\mathrm{B}$ and Conjecture $\mathrm{D}$ (using the notation of [3]).

Perhaps of independent interest is the Corollary to Lemma 3.2 of $\S 3$. It states that there is a homomorphism of the fundamental group of a closed, orientable surface of genus $n$ onto a free group of rank $k$ iff $k \leqq n$.

The author wishes to thank D. R. McMillan, Jr. who suggested the problem. Conversations with Professors McMillan and J. Martin were helpful in the preparation of this announcement.

2. Notation and definitions. The term map is used to mean continuous function. If $f$ is a map from $\left(S, s_{0}\right)$ to $\left(X, x_{0}\right)$, then the homomorphism of $\pi_{1}\left(S, s_{0}\right)$ to $\pi_{1}\left(X, x_{0}\right)$ induced by $f$ is denoted $f_{*}$. Suppose $l$ is a map of $S^{1}$ into a pathwise connected space $S$. Then $l$

${ }^{1}$ Research partially supported by NSF GP-7085 and Wisconsin Graduate Research Committee-WARF Funds. 
defines a conjugate class of elements of $\pi_{1}\left(S, s_{0}\right)$. This class is denoted by $\{l\}$. For any normal subgroup $N$ of $\pi_{1}\left(S, s_{0}\right)$, the statement $\{l\} \in N(\{l\} \notin N)$ is well defined.

A 3-manifold-with-boundary is called a cube-with-handles of genus $n \geqq 0$ if it is orientable and a regular neighborhood of a finite, connected graph with Euler characteristic $1-n$. Let $M$ be a closed, orientable 3-manifold. It is known that $M=U \cup V$ where $U$ and $V$ are cubes-with-handles and $U \cap V$ is their common boundary. The pair $(U, V)$ is called a Heegaard splitting of $M$. The genus of $(U, V)$ is defined to be the genus of $S=\mathrm{Bd} U=\mathrm{Bd} V$. Choose $s_{0} \in S$. The inclusion maps of $S$ into $U$ and $V$ induce homomorphisms $u_{*}$ and $v_{*}$ of $\pi_{1}\left(S, s_{0}\right)$ onto $\pi_{1}\left(U, s_{0}\right)$ and $\pi_{1}\left(V, s_{0}\right)$ respectively. The homomorphism

$$
u_{*} \times v_{*}: \pi_{1}\left(S, s_{0}\right) \rightarrow \pi_{1}\left(U, s_{0}\right) \times \pi_{1}\left(V, s_{0}\right)
$$

is called the splitting homomorphism of $\pi_{1}\left(S, s_{0}\right)$ induced by $(U, V)$.

Suppose $\eta_{1} \times \eta_{2}: \pi_{1}\left(S, s_{0}\right) \rightarrow F_{1} \times F_{2}$ and $\mu_{1} \times \mu_{2}: \pi_{1}\left(S, s_{0}\right) \rightarrow G_{1} \times G_{2}$ are splitting homomorphisms of $\pi_{1}\left(S, s_{0}\right)$. Then $\eta_{1} \times \eta_{2}$ is said to be equivalent to $\mu_{1} \times \mu_{2}$ if there are isomorphisms $\alpha_{1}$ of $F_{1}$ onto $G_{1}$ and $\alpha_{2}$ of $F_{2}$ onto $G_{2}$ so that the diagram

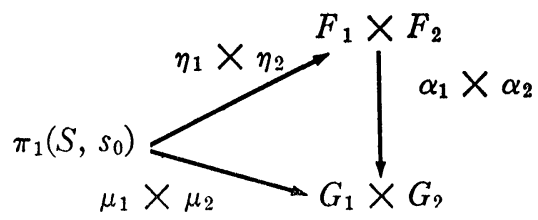

commutes.

3. Mapping surfaces into wedges. The details of the proofs for the lemmas of this section will appear elsewhere. The next few paragraphs give notation which will be used for these lemmas.

Suppose $f$ is a piecewise linear (PL) map of the closed, orientable 2-manifold $S$ into $T$, a wedge at $t_{0}$ of $k$ simple closed curves $X_{1}, \cdots$, $X_{k}$. Suppose $f\left(s_{0}\right)=t_{0}$. For each $i, 1 \leqq i \leqq k$, choose $x_{i} \in X_{i}-\left\{t_{0}\right\}$ which is not a vertex in the subdivision of $T$ for which $f$ is simplicial. Then each component of $f^{-1}\left(x_{i}\right)$ is a polyhedral simple closed curve in $S$. Let $J_{1}$ and $J_{2}$ be distinct components of $f^{-1}\left(x_{j}\right)$. Suppose that $A$ is a polyhedral arc in $S-\left\{s_{0}\right\}$ from $J_{1}$ to $J_{2}$ such that $A \cap \bigcup_{i=1}^{k} f^{-1}\left(x_{i}\right)$ $=\mathrm{Bd} A$.

Let $Q_{1} \subset J_{1}$ and $Q_{2} \subset J_{2}$ be arbitrarily small arcs each containing an endpoint of $A$ in its interior. Let $Q$ denote a small regular neighborhood of $A$ which meets $\cup_{i=1}^{k} f^{-1}\left(x_{i}\right)$ in $Q_{1}$ and $Q_{2}$ each of which is contained in $\mathrm{Bd} Q$. Then the closure of $\mathrm{Bd} Q-\left(Q_{1} \cup Q_{2}\right)$ is two disjoint $\operatorname{arcs} A_{1}$ and $A_{2}$. Both $A_{1}$ and $A_{2}$ are $\operatorname{arcs}$ from $J_{1}$ to $J_{2}$. 
Let $I$ denote the unit interval and let $U\left(x_{i}\right)$ denote an arc in $X_{i}-t_{0}$ with $x_{i}$ in its interior. Then $U\left(x_{i}\right)=x_{i} \times I$ has a product structure with $x_{i}=x_{i} \times \frac{1}{2}$. Let $J$ be a polyhedral simple closed curve in $S$. Then a neighborhood, $U(J)$, of $J$ is said to have a product structure if $U(J)$ $=J \times I$ with $J=J \times \frac{1}{2}$. A map $f$ of $S$ into $T$ will be called transverse with respect to $\bigcup_{i=1}^{k}\left\{x_{i}\right\}$ if each component of $f^{-1}\left(x_{i}\right)$ is a simple closed curve, there exists $U\left(x_{i}\right)$ as above such that each component of $f^{-1}\left(U\left(x_{i}\right)\right)$ has a product structure, and $f$ maps each fiber of $f^{-1}\left(U\left(x_{i}\right)\right)$ homeomorphically onto a fiber of $U\left(\dot{x}_{i}\right)$.

Lemma 3.1. If $f$ maps the arc $A$ into the trivial loop of $T$ based at $x_{j}$, then there is a PL map $g$ of $S$ into $T$ so that

(i) $g$ is homotopic to $f$ keeping $s_{0}$ fixed,

(ii) $g^{-1}\left(x_{i}\right)=f^{-1}\left(x_{i}\right), \quad i \neq j$,

(iii) $g^{-1}\left(x_{j}\right)=f^{-1}\left(x_{j}\right) \cup\left(A_{1} \cup A_{2}\right)-\left(Q_{1} \cup Q_{2}\right)$, and

(iv) $g$ is transverse with respect to $\bigcup_{i=1}^{k}\left\{x_{i}\right\}$.

Lemma 3.2. Let $S$ be a closed, orientable 2-manifold of genus $n>0$. Suppose $f$ is a map of $S$ into $T$, a wedge of $k$ simple closed curves $X_{1}, \cdots, X_{k}$ at $t_{0}$ and $f\left(s_{0}\right)=t_{0}$. If $f_{*}$ is an epimorphism, then there is a PL map $g$ of $S$ into $T$ so that

(i) $g$ is homotopic to $f$ keeping $s_{0}$ fixed,

(ii) for each $i, 1 \leqq i \leqq k$, there is a point $x_{i} \in X_{i}-\left\{t_{0}\right\}$ so that $g^{-1}\left(x_{i}\right)$ is a single simple closed curve $J_{i}$ in $S$,

(iii) $S-\cup_{i=1}^{k} J_{i}$ is connected, and

(iv) $g$ is transverse with respect to $\bigcup_{i=1}^{k}\left\{x_{i}\right\}$.

The proof of Lemma 3.2 uses the notion of "binding-tie" introduced by $\mathrm{J}$. Stallings in [2]. Although, here a more delicate argument is required since the domain of $f$ is the 2-manifold $S$.

An interesting corollary of Lemma 3.2 is

COROLlary 3.3. There is a homomorphism of the fundamental group of a closed, orientable surface of genus $n$ onto a free group of rank $k$ if and only if $k \leqq n$.

Lemma 3.4. Let $S$ be a closed, orientable surface of genus $n>0$. Suppose $f$ is a map of $S$ into $T$, a wedge of $n$ simple closed curves at $t_{0}$ and $f\left(s_{0}\right)=t_{0}$. If $f_{*}$ is an epimorphism, then there is a PL map $g$ of $S$ into $T$ so that

(i) $g$ is homotopic to $f$ keeping $s_{0}$ fixed, and

(ii) the mapping cylinder of $g$ is topologically equivalent to a cubewith-handles of genus $n$. 


\section{Constructing three-manifolds.}

TheOREM 4.1. Let $S$ be a closed, orientable 2-manifold of genus $n>0$. Suppose $\eta_{1} \times \eta_{2}$ is a splitting homomorphism of $\pi_{1}\left(S, s_{0}\right)$ into $F_{1} \times F_{2}$. Then there is a closed, orientable 3-manifold $M$ and a Heegaard splitting $(U, V)$ of $M$ so that the splitting homomorphism induced by $(U, V)$ is equivalent to $\eta_{1} \times \eta_{2}$.

Proof. For $k=1,2$, let $T_{k}$ be a wedge at $t_{k}$ of $n$ simple closed curves. Identify $\pi_{1}\left(T_{k}, t_{k}\right)$ with $F_{k}$ and let $f_{k}$ be a map of $\left(S, s_{0}\right)$ into $\left(T_{k}, t_{k}\right)$ so that $\left(f_{k}\right)_{*}=\eta_{k}$. From Lemma 3.4 and for $k=1,2$, there is a PL map $g_{k}$ of $\left(S, s_{0}\right)$ into $\left(T_{k}, t_{k}\right)$ so that $g_{k}$ is homotopic to $f_{k}$ keeping $s_{0}$ fixed and the mapping cylinder $C\left(g_{k}\right)$ of $g_{k}$ is a cube-with-handles of genus $n$.

Let $M$ be the closed three-manifold obtained by considering $C\left(g_{1}\right) \cup C\left(g_{2}\right)$ as a disjoint union and identifying $s \in S=\operatorname{Bd} C\left(g_{1}\right)$ with $s \in S=\mathrm{Bd} C\left(g_{2}\right)$.

There is an isomorphism $\alpha_{k}$ of $\pi_{1}\left(C\left(g_{k}\right), s_{0}\right)$ onto $\pi_{1}\left(T_{k}, t_{k}\right)$ so that if $e_{k}$ is the inclusion of $S$ into $C\left(g_{k}\right)$, then $\alpha_{k}\left(e_{k}\right)_{*}=\left(f_{k}\right)_{*}$. It follows that the diagram

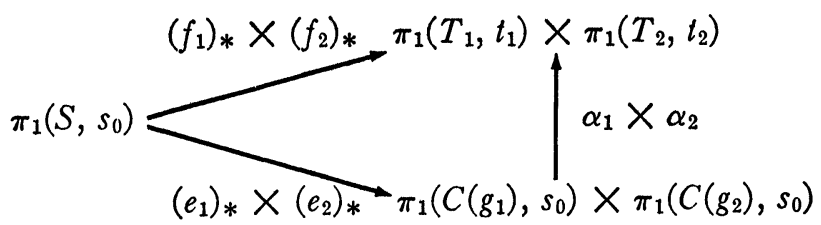

commutes. Let $U=C\left(g_{1}\right)$ and $V=C\left(g_{2}\right)$. The splitting homomorphism induced by $(U, V)$ is $\left(e_{1}\right)_{*} \times\left(e_{2}\right)_{*}$. Since $\left(f_{1}\right)_{*} \times\left(f_{2}\right)_{*}=\eta_{1} \times \eta_{2}$ the theorem is proved.

The notation $G_{1} * G_{2}$ is used for the free product of the groups $G_{1}$ and $G_{2}([1$, Vol. II, p. 11]).

In [3], J. Stallings states the following conjectures:

Conjecture B. Let $S$ be a closed, orientable 2-manifold of genus $n>1$. Let $F_{1}$ and $F_{2}$ be free groups of rank $n$. Let $\eta: \pi_{1}(S) \rightarrow F_{1} \times F_{2}$ be an epimorphism. Then there is a nontrivial element $\{l\} \in$ kernel $\eta$ where $l$ is a simple closed curve on $S$.

Conjecture D. In the situation of Conjecture B, the map $\eta: \pi_{1}(S)$ $\rightarrow F_{1} \times F_{2}$ can be factored through an essential map of $\pi_{1}(S)$ into some free product $G_{1} * G_{2}$.

A homomorphism $\phi$ of a group $G$ into a free product $G_{1} * G_{2}$ is essential if there is no element $g \in G_{1} * G_{2}$ such that $g \phi(G) g^{-1}$ is contained in one of the factors $G_{1}$ or $G_{2}$. 
Theorem 4.2. Conjecture B is true if and only if Poincarê's Conjecture is true if and only if Conjecture $\mathrm{D}$ is true.

Proof. It is shown in [3] that Conjecture B implies Poincaré's Conjecture.

It is now shown that Poincaré's Conjecture implies Conjecture D. Let $\eta=\eta_{1} \times \eta_{2}$ be the epimorphism given in Conjecture D. Let $M$ denote the 3-manifold with Heegaard splitting $(U, V)$ guaranteed in Theorem 4.1 corresponding to the splitting homomorphism $\eta_{1} \times \eta_{2}$ of $\pi_{1}\left(S, s_{0}\right)$ into $F_{1} \times F_{2}$. Then $M$ is simply connected, since $\eta$ is an epimorphism (Theorem 1 of [3]). Thus by Poincaré's Conjecture, $M$ is the 3-sphere $S^{3}$. The Heegaard splitting $(U, V)$ of $S^{3}$ has genus $n>1$. By Waldhausen [4], there is a simple closed curve $l$ in $S,\{l\} \neq 1$ in $\pi_{1}(S)$. Also, if $u_{*} \times v_{*}$ is the splitting homomorphism induced by $(U, V)$, then $\{l\} \in$ kernel $u_{*} \cap$ kernel $v_{*}$, and $l$ separates $S$. To factor $\eta$ through a free product, a wedge $S^{\prime}$ is formed from $S$ by identifying the simple closed curve $l$ to a point. The fundamental group of $S^{\prime}$ is the desired nontrivial free product.

From Theorem 2 of [3], Conjecture D implies Conjecture B.

REMARK. The author has shown, with appropriate definitions for equivalence, that there is a one-one correspondence between equivalence classes of splitting-homomorphisms and equivalence classes of Heegaard splittings.

\section{REFERENCES}

1. A. G. Kurosh, The theory of groups, 2nd ed., Vol. II, Chelsea, New York, 1960.

2. J. Stallings, A topological proof of Grushko's theorem on free products, Math. Z. 90 (1965), 1-8.

3. - How not to prove the Poincare conjecture, Topology Seminar, Wisconsin, 1965, Princeton Univ. Press, Princeton, N. J.

4. Friedhelm Waldhausen, Heegaard-Zerlegungen der 3-Sphare, Topology 7 (1968), 195-203.

UNIVERSITY OF WisCONSIN 